\title{
BEM AND FEM RESULTS OF DISPLACEMENTS IN A POROELASTIC COLUMN
}

\author{
Bettina AlberS *, Stavros A. SAVIDiS *, H. Ercan TAŞAN **, Otto von EStorfF ***, \\ MALTE GEHLKEN *** \\ * Institute of Geotechnical Engineering and Soil Mechanics \\ Berlin Institute of Technology (TU Berlin), Sekr. TIB1-B7, Gustav-Meyer-Allee 25, D-13355 Berlin, Germany \\ e-mail:albers@grundbau.tu-berlin.de
}

** TRADYNA GmbH, Spandauer Damm 57, D-14059 Berlin, Germany

*** Institute of Modelling and Computation

Hamburg University of Technology (TUHH), Denickestrasse 17, D-21073 Hamburg, Germany

\begin{abstract}
The dynamical investigation of two-component poroelastic media is important for practical applications. Analytic solution methods are often not available since they are too complicated for the complex governing sets of equations. For this reason, often some existing numerical methods are used. In this work results obtained with the finite element method are opposed to those obtained by Schanz using the boundary element method. Not only the influence of the number of elements and time steps on the simple example of a poroelastic column but also the impact of different values of the permeability coefficient is investigated.
\end{abstract}

Keywords: finite element method, boundary element method, poroelastic media.

\section{Introduction}

The initiative for the present comparison of results of the Finite Element Method (FEM) and the Boundary Element Method (BEM) is a project in which both the methods are used and, finally, FEM and BEM subdomains will be coupled iteratively (for information about iterative coupling, see the work of von Estorff and Hagen (2006)). The propagation of sound and vibrations due to bankside shipping traffic is the topic of this project. Two paths of sound and vibrations are of interest: the propagation from the ship through water and air to the immission place (this path is investigated using the Fast Multipole Boundary Element Method (Fischer, 2004)), and the propagation through the subsoil to the construction (this path is examined using a two-component poroelastic model which is implemented into a finite element program (Savidis et al., 2011)). This FEM model is used in the present paper for the comparison with the BEM results obtained by Schanz (2001). In both approaches the displacements in a column consisting of a porous material are considered.
1.1. Theories for saturated porous media. In soil mechanics the ground is often considered a one-component elastic solid. However, this is not sufficient to investigate the flow of a fluid through the soil particles. If the latter is accounted for, the soil needs to be considered a two- or even three-component, so-called porous or granular medium. Such a medium consists of the skeleton and at least one pore fluid. If the solid particles are held together by compression or cementing material, one speaks of porous media, if they are loose, one denotes them as a granular material. The investigation of such materials looks back on a tradition of approximately 70 years.

The first, and probably most often applied model is the Biot model. It was first introduced in 1941 by Maurice A. Biot for the investigation of consolidation problems and later for the description of the wave propagation in porous media (Biot, 1956). During the ensuing period, after applying his model to wave propagation himself, the model was used and modified by various authors for the investigation of several applications. Moreover, further models were developed in order to describe various 
processes (also of nonlinear type) in porous and granular media, e.g., the Goodman and Cowin theory (Goodman and Cowin, 1972), TPM (Theory of Porous Media) (de Boer and Ehlers, 1986) or the Wilmanski model (Wilmanski, 1996). Initially, these models were analyzed analytically or half analytically (numerical interpretation of an analytical solution).

Since the 1950s/1960s numerical methods have rapidly developed, first the finite element method, then the boundary element method and, finally, also further progresses. In order to use such methods also for porous media and to minimize the computing time, it became necessary to simplify the complex systems of equations for porous media (various partial balance equations for several components). For this purpose, another form of the Biot model arose, developed by Zienkiewicz and Shiomi (1984). Such a modification of the Biot model will be used for the investigations in this work. Therein, the principle of effective stresses (von Terzaghi, 1936) is used and the fluid velocity is described by the Darcy law. Then, the relative velocity between the two components, fluid and solid, is identified by the filter velocity. Thereby, one kinematic becomes lost, which means that the second (slower) longitudinal wave which has been predicted in the saturated porous medium by both the Biot and Wilmanski models cannot be observed. However, for practical applications this does not play an important role since this wave is so strongly attenuated that it is hard to provide its evidence in real media. Theoretically, this has been shown by Albers (2010), while information about the experimental verification can be found in the works of Kelder and Smeulders (1997), as well as Plona (1980).

Several authors were engaged in the numerical solution of problems in porous media by the use of the FEM. Panneton and Atalla (1997) used the FEM to solve the three-dimensional poroelasticity problem in acoustics based on the Biot theory modified by Allard (1993). They derived a displacement-based FE model using a Lagrangian approach and set up an analogy with solid elements. The efficient algorithm allows the use of classical FEM codes (Hild, 2011) for poroelastic media. Earlier, Göransson (1995) introduced also a simplified approach. He neglected the elastic coupling between fluid and solid in order to reduce the model to two variables, i.e., solid displacement and pore fluid pressure. However, the focus was not on the efficiency of the numerical implementation. The improvement in the accuracy and effectiveness of such a model was faced by Atalla et al. (1998), who showed the mixed displacement-pressure formulation involving the dynamic equations of the skeleton in vacuo and the equivalent fluid in the rigid skeleton limit together with their weak integral form and discussed the implementation in an FE code. This work was enhanced concerning the weak formulation by Atalla et al. (2001). The approach of
Zienkiewicz et al. (1999), which is also used in the present manuscript, is extended by Lewis and Schrefler (1998) as well as Schrefler and Scotta (2001), e.g., for problems in unsaturated porous media. Korsawe and Starke (2005), as well as Korsawe et al. (2006), made progress in the FE approach of the Biot consolidation problem in mixed formulation, especially in the development of a least-squares FE method.

In the following, first the original form of the Biot model will be introduced. Then it is shown which simplifications yield the modified form of the Biot model used in this work. Afterwards it will be shown how the equations have to be prepared for the use of the FEM and the BEM. The FEM is applied to a simple example which has been investigated before by Schanz (2001) using the BEM. Results are compared with respect to the number of elements, the number of time steps, and the influence of the permeability of the porous material.

1.2. Original form of the Biot model. The Biot model is used to model the subsoil. It consists of two components: the solid grains which build a skeleton $S$ and the pore fluid $F$. The model is presented here first in the version of the original article by Biot (1956).

Most important are the momentum balances given by

$$
\begin{aligned}
N & \nabla^{2} \mathbf{u}+\nabla[(A+N) e+Q \varepsilon] \\
= & \frac{\partial^{2}}{\partial t^{2}}\left(\rho_{11} \mathbf{u}+\rho_{12} \mathbf{U}\right)+b \frac{\partial}{\partial t}(\mathbf{u}-\mathbf{U}), \\
\nabla & {[Q e+R \varepsilon] } \\
& =\frac{\partial^{2}}{\partial t^{2}}\left(\rho_{12} \mathbf{u}+\rho_{22} \mathbf{U}\right)-b \frac{\partial}{\partial t}(\mathbf{u}-\mathbf{U}) .
\end{aligned}
$$

In these equations $\mathbf{u}$ and $\mathbf{U}$ are the displacements of the solid and the fluid, respectively. The volume changes in the solid and in the fluid are denoted by $e$ and $\varepsilon$, respectively. They are related to the displacements by

$$
e=\nabla \cdot \mathbf{u}, \quad \varepsilon=\nabla \cdot \mathbf{U} .
$$

This means that the equations can be expressed also by using only the displacements as variables, which results in

$$
\begin{gathered}
N \nabla^{2} \mathbf{u}+(A+N) \nabla(\nabla \cdot \mathbf{u})+Q \nabla(\nabla \cdot \mathbf{U}) \\
=\frac{\partial^{2}}{\partial t^{2}}\left(\rho_{11} \mathbf{u}+\rho_{12} \mathbf{U}\right)+b \frac{\partial}{\partial t}(\mathbf{u}-\mathbf{U}), \\
Q \nabla(\nabla \cdot \mathbf{u})+R \nabla(\nabla \cdot \mathbf{U}) \\
=\frac{\partial^{2}}{\partial t^{2}}\left(\rho_{12} \mathbf{u}+\rho_{22} \mathbf{U}\right)-b \frac{\partial}{\partial t}(\mathbf{u}-\mathbf{U}) .
\end{gathered}
$$

$A, N$ and $Q$ are material parameters associated with elasticity constants and static coupling. $R$ is connected with the compressibility and the mass density of the 
fluid. Here $\rho_{11}, \rho_{22}$ and $\rho_{12}$ are mass coefficients which are associated with the solid, the fluid and a coupling between the components, respectively. The coefficient $b$ is related to Darcy's coefficient of permeability $k$. The latter has the unit $\left[\mathrm{m}^{3} \mathrm{~s} / \mathrm{kg}\right]$, not as usual in soil mechanics $\left[k_{f}\right]=[\mathrm{m} / \mathrm{s}]$ (permeability coefficient). However, the two quantities are related by $k=k_{f} /\left(\rho_{f} g\right)$, where $\rho_{f}$ and $g$ are the fluid mass density and the earth acceleration, respectively.

1.3. Modified form of the Biot model. For practical applications, it is more reasonable to use, instead of the displacement of the fluid (which is a rather fictive quantity but nevertheless often used) variables which are customary in soil mechanics, e.g., displacements of the solid and the pore water pressure. Moreover, instead of the custom description of stresses in continuum mechanics by partial stresses, i.e., separately for each component, the formulation by means of total stresses is often preferred. Zienkiewicz et al. (1999) introduced such a description and compared different possibilities of simplification. In the following this modified form of the Biot model is presented.

The stresses enter the model not as partial stresses, but as a total stress. By the principle of effective stresses, the stress is split into a part which is taken over by the skeleton and the fluid pressure. It is assumed that the deformation of the skeleton is an intrinsic property which is not influenced by the fluid pressure.

In the framework of the FEM it became common to use a vector description of the stress tensor, the so-called Voigt notation, which reduces the components to six instead of nine, i.e.,

$$
\sigma=\left[\begin{array}{llllll}
\sigma_{x} & \sigma_{y} & \sigma_{z} & \tau_{x y} & \tau_{y z} & \tau_{z x}
\end{array}\right]^{T} .
$$

The stress in the skeleton is defined as the effective stress $\sigma^{\prime}$, i.e., it is transferred from soil particle to soil particle. Simultaneously, the hydrostatic stress is determined by the pore pressure $p$ : It is $-\alpha \mathbf{m} p$, where $\mathbf{m}=\left[\begin{array}{cccccc}1 & 1 & 1 & 0 & 0 & 0\end{array}\right]^{T}$. The parameter $\alpha$ takes into account the deformation of the skeleton. Holler (2006) refers to it as the Biot or the Bishop constant. It is determined by the fraction of the compressibility moduli of the skeleton $K$, and of the single particle $K_{s}$ : $\alpha=1-K / K_{s}$. For Berea sandstone, for instance, which is used in the numerical example in Section 4, $K=8 \cdot 10^{9} \mathrm{~N} / \mathrm{m}^{2}$ and $K_{s}=3.6 \cdot 10^{10} \mathrm{~N} / \mathrm{m}^{2}$ (Schanz and Cheng, 2000), thus $\alpha=0 . \overline{7}$.

Following the common convention, tensile forces yield positive stresses. Thus, the part which concerns the pore pressure has a negative sign. Thereby, the following relation follows between total and effective stresses:

$$
\sigma=\sigma^{\prime}-\alpha \mathbf{m} p
$$

If the compressibility modulus of a single particle is much larger than the one of the whole material, then $\alpha \rightarrow 1$, and one obtains the classical form of the effective stress which has been introduced by von Terzaghi (1936).

The kinematic compatibility describes the relation between displacements $\mathbf{u}$ and strains $\varepsilon$ which (in matrix notation) is given by

$$
\varepsilon=\mathbf{d}_{S} \mathbf{u}
$$

where the displacements $\mathbf{u}=\left[\begin{array}{lll}u_{x} & u_{y} & u_{z}\end{array}\right]^{T}$ and the matrix of derivatives

$$
\mathbf{d}_{S}=\left[\begin{array}{cccccc}
\frac{\partial}{\partial x} & 0 & 0 & \frac{\partial}{\partial y} & 0 & \frac{\partial}{\partial z} \\
0 & \frac{\partial}{\partial y} & 0 & \frac{\partial}{\partial x} & \frac{\partial}{\partial z} & 0 \\
0 & 0 & \frac{\partial}{\partial z} & 0 & \frac{\partial}{\partial y} & \frac{\partial}{\partial x}
\end{array}\right]^{T}
$$

are used. Due to the vectorial description, i.e., Voigt's notation, engineering strains are used.

If now the relative velocity between solid and fluid, $\mathbf{w}=\mathbf{v}_{f}-\mathbf{v}_{s}$, is identified by the Darcy velocity, a momentum balance for the soil-fluid-mixture can be formulated,

$$
\mathbf{d}_{S}^{T} \sigma-\rho \ddot{\mathbf{u}}-\rho_{f}\left[\dot{\mathbf{w}}+\mathbf{w} \nabla^{T} \mathbf{w}\right]+\rho \mathbf{b}=0,
$$

with

$$
\dot{\mathbf{w}}=\frac{\mathrm{d} \mathbf{w}}{\mathrm{d} t} .
$$

Here, $\rho_{f}$ is the mass density of the fluid, $\mathbf{b}$ is the vector of the body force per unit mass (commonly the gravitation) and $\rho$ is the mass density of the total body, defined as

$$
\rho=n \rho_{f}+(1-n) \rho_{s}
$$

where the soil particles have the mass density $\rho_{s}$ and $n$ is the porosity, i.e., the volume of the voids over the total volume.

The term $\rho_{f}[\dot{\mathbf{w}}+\mathbf{w} \nabla \mathbf{w}]$ reflects the fluid acceleration relative to the solid and its convective parts. However, in general it is small and therefore will be neglected further on. Thus, one ends up with

$$
\mathbf{d}_{S}^{T} \sigma-\rho \ddot{\mathbf{u}}+\rho \mathbf{b}=0 .
$$

Another balance law is stated for the fluid, again, assuming that the fluid moves together with the solid. This means that the relative velocity of the components is identified with the filter velocity. The balance law reads

$$
-\nabla p-\mathbf{R}-\rho_{f} \ddot{\mathbf{u}}-\frac{\rho_{f}\left[\dot{\mathbf{w}}+\mathbf{w} \nabla^{T} \mathbf{w}\right]}{n}+\rho_{f} \mathbf{b}=0,
$$

where $\mathbf{R}$ denotes viscous drag forces which, by use of Darcy's law, can be expressed by $\mathbf{k R}=\mathbf{w}$. 
The convective part in Eqn. (10), again, is small and also the contribution of the solid acceleration can be neglected. Its inclusion would cause the final equation to be non-symmetric (see Zienkiewicz et al., 1999). For these reasons, (10) is converted to

$$
\mathbf{w}=k\left(-\nabla p+\rho_{f} \mathbf{b}\right),
$$

with the permeability parameter $k$ with dimensions [length $]^{3}[$ time $] /[$ mass $]$.

The third and last equation is the mass balance of the flow. It has the form

$$
\nabla^{T} \mathbf{w}+\alpha \mathbf{m} \dot{\varepsilon}+\frac{\dot{p}}{Q}+\underbrace{n \frac{\dot{\rho}_{f}}{\rho_{f}}}_{\text {small }}+\underbrace{\dot{s}_{0}}_{\text {not relevant }}=0,
$$

where

$$
\frac{1}{Q}=\frac{n}{K_{f}}+\frac{\alpha-n}{K_{s}}
$$

with $K_{s}$ being the compressibility modulus of the solid, $K_{f}$ the compressibility modulus of the fluid and $n$ the initial porosity. Their values for Berea sandstone are given in Table 1. The last two parts $n \frac{\dot{\rho}_{f}}{\rho_{f}}$ and $\dot{s}_{0}$ of Eqn. (12) represent the change in the mass density and the volumetric expansion of the solid in the case of thermal changes. Since these are not considered in this manuscript, the latter contribution can be omitted. Referring to Zienkiewicz et al. (1999), who provide estimates of the contributions to Eqn. (12), also the former is left out since it is small in comparison with the other terms of (12).

Equations (9), (11) and (12) build the simplified set of equations for the unknown variables $p \equiv p_{f}$, w, and $\mathbf{u}$, which are the fluid pressure, the fluid velocity and the displacements of the solid, respectively.

1.4. u-p formulation of the model. For numerical calculations, it has become common to further simplify the system (see Zienkiewicz et al., 1999). On the one hand, the above-mentioned negligible quantities are omitted, on the other, the variable $\mathrm{w}$ is eliminated so that only two variables are left. This form of the equations is called u-p formulation. It consists of Eqn. (9) and the combination of (11) and (12),

$$
\nabla^{T} \mathbf{k}\left(-\nabla p+\rho_{f} \mathbf{b}\right)+\alpha \mathbf{m} \mathbf{d}_{S} \dot{\mathbf{u}}=0 .
$$

Zienkiewicz et al. (1999) state that this reduced system suffers from a loss of accuracy only for problems containing high frequency oscillations.

\section{Preparation for the use of finite elements}

The system of equations derived above has been investigated in different versions for several applications.
Table 1. Material parameters of Berea sandstone (after Schanz and Cheng, 2000).

\begin{tabular}{|l||l|}
\hline Initial porosity & $n=0.19$ \\
\hline Compressibility modulus of solid & $K_{s}=3.6 \cdot 10^{10} \mathrm{~N} / \mathrm{m}^{2}$ \\
\hline Compressibility modulus of fluid & $K_{f}=3.3 \cdot 10^{9} \mathrm{~N} / \mathrm{m}^{2}$ \\
\hline Bulk compressibility modulus & $K=8 \cdot 10^{9} \mathrm{~N} / \mathrm{m}^{2}$ \\
\hline
\end{tabular}

Naumann (2004) and Savvidis (2009) derived the model in its two-dimensional form from basic equations and implemented it in the finite element package ANSYS. In the work of Rackwitz et al. (2005), the element for coupled fluid-structure calculations was used to investigate quasi-static processes. In particular, the transient behavior and the development of the pore water pressure were examined. A further development of the model is shown by Taşan et al. (2010) and Taşan (2012), who show the three-dimensional version used for the dynamic investigation of monopile offshore foundations. In these works also a hypoplastic behavior of the soil has been taken into account. Plans exist to use the two-phase element also for rail track subsoil systems (cf. Kogut and Ciurej, 2010).

The system (9), (13) is now used to implement a two-phase element into ANSYS. For this purpose, first the weak formulation of these equations is built. This is performed by the weighted residual method, i.e., a solution statement is sought, which satisfies a differential equation $\mathrm{d} f(x)=0$ at each point of the system. However, an exact analytic solution can only be found for simple systems. For more complicated systems one has to rely on approximate solutions. Since these solutions do not satisfy the differential equation in each point, a residuum $r$ remains, i.e., $\mathrm{d} f(x)=r$. The weighted residual method is a procedure to minimize this remainder. It is required that the integral of the residuum, which includes a weighting function $\tilde{g}$, over the investigated domain $G$, $\int_{G} \tilde{g} \mathrm{~d} f(x) \mathrm{d} G$, vanish. After integration, a spatial and a temporal discretization of the equations are carried out.

\subsection{Weak formulation and discretization of the solid.} The weak formulation of the equation for the solid (9) is

$$
\begin{aligned}
\int_{V} \tilde{\mathbf{g}}^{T}\left(\mathbf{d}_{S}^{T} \sigma^{\prime}-\alpha \mathbf{d}_{S}^{T} \mathbf{m} p\right) \mathrm{d} V \\
\quad-\int_{V} \tilde{\mathbf{g}}^{T} \rho \ddot{\mathbf{u}} \mathrm{d} V+\int_{V} \tilde{\mathbf{g}}^{T} \rho \mathbf{b} d V=0 .
\end{aligned}
$$

Trial functions $\mathbf{N}$ and $\mathbf{N}_{p}$ are chosen to approximate the displacement, the velocity, the acceleration, and the pore water pressure, which leads to

$$
\mathbf{u}=\mathbf{N} \mathbf{u}_{k}, \quad \dot{\mathbf{u}}=\mathbf{N} \dot{\mathbf{u}}_{k}, \quad \ddot{\mathbf{u}}=\mathbf{N} \ddot{\mathbf{u}}_{k}, \quad p=\mathbf{N}_{p} \mathbf{p}_{k} .
$$


Moreover, the weighting function is chosen as a variation of the displacement $\mathbf{u}$. According to the Galerkin method, for the weighting function $\tilde{\mathrm{g}}^{T}$ the same trial function is used as for the displacement,

$$
\tilde{\mathbf{g}}^{T}=\delta \mathbf{u}^{T}=\delta\left(\mathbf{N} \mathbf{u}_{k}\right)^{T}=\delta \mathbf{u}_{k}^{T} \mathbf{N}^{T} .
$$

A surface term (surface $R$ ) enters the equation due to application of the Gauss theorem to the first term of Eqn. (14). By substituting (15) into (14) the governing equation

$$
\begin{aligned}
& \int_{R} \delta \mathbf{u}_{k}^{T} \mathbf{N}^{T} \sigma_{R} \mathbf{n} \mathrm{d} R-\int_{V} \delta \mathbf{u}_{k}^{T} \mathbf{N}^{T} \mathbf{d}_{S}^{T} \sigma^{\prime} \mathrm{d} V \\
& +\int_{V} \delta \mathbf{u}_{k}^{T} \mathbf{N}^{T} \mathbf{d}_{S}^{T} \mathbf{m} \mathbf{N}_{p} \mathbf{p}_{k} \mathrm{~d} V \\
& \quad-\int_{V} \delta \mathbf{u}_{k}^{T} \mathbf{N}^{T} \rho \mathbf{N} \ddot{\mathbf{u}}_{k} \mathrm{~d} V+\int_{V} \delta \mathbf{u}_{k}^{T} \mathbf{N}^{T} \rho \mathbf{b} \mathrm{d} V=0,
\end{aligned}
$$

is obtained, where the total stress on the surface $\sigma_{R}$ consists of two parts $\sigma_{R}^{\prime}$ and $\alpha \mathbf{m} p_{R}$ corresponding to the effective stress and the pore pressure on the surface.

Since the nodal values of the displacements $\mathbf{u}_{k}$, the velocities $\dot{\mathbf{u}}_{k}$, the accelerations $\ddot{\mathbf{u}}_{k}$ as well as the variations in the nodal values $\delta \mathbf{u}_{k}$ are constant, they can be extracted from the integral. Furthermore, the matrix of the derivatives of trial functions $\mathbf{B}=\mathbf{d}_{S} \mathbf{N}$ and the matrix of trial functions for the pore water pressure $\mathbf{N}_{p}=\mathbf{m} \mathbf{N}_{p}^{T}$ are introduced and

$$
\mathbf{M}:=\int_{V} \mathbf{N}^{T} \rho \mathbf{N} \mathrm{d} V
$$

the mass matrix,

$$
\mathbf{Q}:=\int_{V} \mathbf{B}^{T} \mathbf{N}_{p} \mathrm{~d} V
$$

the coupling matrix,

$$
\mathbf{f}_{R}^{s}:=\int_{R} \mathbf{N}^{T} \sigma_{R} \mathrm{~d} R
$$

the load vector of the the surface forces from outside, and $\mathbf{f}_{g}^{s}:=\int_{V} \mathbf{N}^{T} \rho \mathbf{b} d V$, the load vector of the net weight, are defined. The sum $\mathbf{f}_{R}^{s}+\mathbf{f}_{g}^{s}$ forms the load vector $\mathbf{f}^{s}$ of the solid. After exclusion of the variations $\delta \mathbf{u}_{k}$ and insertion of the linear elastic constitutive law, one obtains

$$
\mathbf{M} \ddot{\mathbf{u}}_{k}+\mathbf{K}_{E}^{s} \mathbf{u}_{k}-\mathbf{Q} \mathbf{p}_{k}=\mathbf{f}^{s},
$$

where

$$
\mathbf{K}_{E}^{s}:=\int_{V} \mathbf{B}^{T} \mathbf{E B} \mathrm{d} V
$$

denotes the structural stiffness matrix in the case of linear elasticity. This means that the effective stress is $\sigma^{\prime}=$ $\mathbf{E} \varepsilon=\mathbf{E d}_{S} \mathbf{N u}=\mathbf{E B u}$ with the elasticity matrix

$$
\mathbf{E}=\frac{E}{1+\nu}\left[\begin{array}{cccccc}
\frac{1-\nu}{1-2 \nu} & \frac{\nu}{1-2 \nu} & \frac{\nu}{1-2 \nu} & 0 & 0 & 0 \\
\frac{\nu}{1-2 \nu} & \frac{1-\nu}{1-2 \nu} & \frac{\nu}{1-2 \nu} & 0 & 0 & 0 \\
\frac{\nu}{1-2 \nu} & \frac{\nu}{1-2 \nu} & \frac{1-\nu}{1-2 \nu} & 0 & 0 & 0 \\
0 & 0 & 0 & \frac{1}{2} & 0 & 0 \\
0 & 0 & 0 & 0 & \frac{1}{2} & 0 \\
0 & 0 & 0 & 0 & 0 & \frac{1}{2}
\end{array}\right]
$$

containing the Poisson number $\nu$.

\subsection{Weak formulation and discretization of the fluid.} The water pressure has no direction, therefore in the case of the fluid the weighting function $\tilde{g}$ is a scalar. After weighting and integrating Eqn. (13), the weak formulation

$$
\begin{aligned}
& \frac{1}{\rho_{f} g} \int_{V} \tilde{g} \nabla \mathbf{k}\left(-\nabla p+\rho_{f} \mathbf{b}\right) \mathrm{d} V \\
& \quad+\alpha \int_{V} \tilde{g} \mathbf{m}^{T} \frac{\partial}{\partial t}\left(\mathbf{d}_{S} \mathbf{u}\right) \mathrm{d} V=0 .
\end{aligned}
$$

is obtained.

The weighting function $\tilde{g}$ is chosen to be a variation in the pore water pressure $p$, i.e., $\tilde{g}=\delta p$. Insertion into the weak formulation, transformation by partial integration, and application of the Gauss theorem yields

$$
\begin{aligned}
& \frac{1}{\rho_{f} g} \int_{R} \mathbf{n}^{T} \delta p \mathbf{k}\left(-\nabla p+\rho_{f} \mathbf{b}\right) \mathrm{d} R \\
& +\frac{1}{\rho_{f} g} \int_{V} \delta p \nabla \mathbf{k}\left(\nabla p-\rho_{f} \mathbf{b}\right) \mathrm{d} V \\
& +\alpha \int_{V} \delta p \mathbf{m}^{T} \frac{\partial}{\partial t}\left(\mathbf{d}_{S} \mathbf{u}\right) \mathrm{d} V=0,
\end{aligned}
$$

where $\mathbf{n}$ is the unit vector of the surface.

The use of

$$
\mathbf{v}_{f}=\frac{1}{\rho_{f} g} \mathbf{k}\left(-\nabla p+\rho_{f} \mathbf{b}\right),
$$

the definition of the flow of the pore water perpendicular to the surface $q=\mathbf{n}^{T} \mathbf{v}_{f}$, and the trial functions for displacement as well as pore water pressure (Eqn. 15) results in

$$
\begin{aligned}
\int_{R} \delta \mathbf{p}_{k}^{T} \mathbf{N}_{p} q \mathrm{~d} R \\
\quad+\frac{1}{\rho_{f} g} \int_{V} \delta \mathbf{p}_{k}^{T} \mathbf{N}_{p} \nabla \mathbf{k}\left(\nabla \mathbf{N}_{p}^{T} \mathbf{p}_{k}-\rho_{f} \mathbf{b}\right) \mathrm{d} V \\
\quad+\alpha \int_{V} \delta \mathbf{p}_{k}^{T} \mathbf{N}_{p} \mathbf{m}^{T} \mathbf{d}_{S} \mathbf{N} \dot{\mathbf{u}}_{k} \mathrm{~d} V=0 .
\end{aligned}
$$


The term

$$
\mathbf{f}_{R}^{p}:=-\int_{R} \mathbf{N}_{p} q \mathrm{~d} R
$$

describes the flow of the pore water at the surface of the element and distributes the surface flow on the surface nodes via the form function vector of the pore water pressure $\mathbf{N}_{p}$. The negative sign shows that the positive direction of the pore water flow $q$ is directed outwards. In the second term of (21) the matrix of derivatives of trial functions concerning the pore water pressure $\mathbf{B}_{p}=$ $\nabla \mathbf{N}_{p}^{T}$, the permeability matrix

$$
\mathbf{H}=\frac{1}{\rho_{f} g} \int_{V} \mathbf{B}_{p}^{T} \mathbf{k B}_{p} \mathrm{~d} V,
$$

and the volume load vector of the fluid

$$
\mathbf{f}_{g}^{p}=\frac{1}{g} \int_{V} \mathbf{B}_{p}^{T} \mathbf{k b ~ d} V
$$

are introduced. The permeability of the soil is taken into account by $\mathbf{H}$. It distributes the permeability properties on the nodes via matrix $\mathbf{B}_{p}$. The vector containing the degrees of freedom of the pore water pressure $\mathbf{p}_{k}$ and also its variations $\delta \mathbf{p}_{k}$ can be excluded from the integral. With the definition of the load vector for the fluid, $\mathbf{f}^{p}=\mathbf{f}_{R}^{p}+\mathbf{f}_{g}^{p}$, the equation for the fluid is obtained,

$$
\mathbf{Q}^{T} \dot{\mathbf{u}}_{k}+\mathbf{H} \mathbf{p}_{k}=\mathbf{f}^{p}
$$

2.3. Governing equations. Summarizing the last two subsections, the complete set of equations for the finite element, which can be used for linear elastic coupled fluid-structure calculations, is given by

$$
\begin{gathered}
{\left[\begin{array}{cc}
\mathbf{M} & \mathbf{0} \\
\mathbf{0} & \mathbf{0}
\end{array}\right]\left(\begin{array}{c}
\ddot{\mathbf{u}}_{k} \\
\ddot{\mathbf{p}}_{k}
\end{array}\right)+\left[\begin{array}{cc}
\mathbf{0} & \mathbf{0} \\
\mathbf{Q}^{T} & \mathbf{0}
\end{array}\right]\left(\begin{array}{c}
\dot{\mathbf{u}}_{k} \\
\dot{\mathbf{p}}_{k}
\end{array}\right)} \\
+\left[\begin{array}{cc}
\mathbf{K}_{E}^{s} & -\mathbf{Q} \\
\mathbf{0} & \mathbf{H}
\end{array}\right]\left(\begin{array}{l}
\mathbf{u}_{k} \\
\mathbf{p}_{k}
\end{array}\right)=\left(\begin{array}{c}
\mathbf{f}^{s} \\
\mathbf{f}^{p}
\end{array}\right) .
\end{gathered}
$$

Here, $\mathbf{M}$ is the mass matrix, $\mathbf{H}$ is the permeability matrix, $\mathbf{Q}$ is the coupling matrix, $\mathbf{K}_{E}^{s}$ is the stiffness matrix of the structure, and $\mathbf{f}^{s}$ and $\mathbf{f}^{p}$ are the load vectors of the solid and the fluid, respectively.

2.4. Temporal discretization. To solve the set of equations (23), a time step procedure-the Newmark method is used (see, e.g. Taşan, 2012). It is an implicit integration procedure for the solution of differential equations of second order and has at time $t_{n+1}$ the following form:

$$
\mathbf{M} \ddot{\mathbf{u}}_{n+1}+\mathbf{C} \dot{\mathbf{u}}_{n+1}+\mathbf{K} \mathbf{u}_{n+1}=\mathbf{f} .
$$

Using the known initial conditions for the displacements $\mathbf{u}_{n}$, velocities $\dot{\mathbf{u}}_{n}$ and accelerations $\ddot{\mathbf{u}}_{n}$ at time $t_{n}$, the quantities at time $t_{n+1}$ are calculated:

$$
\begin{aligned}
\dot{\mathbf{u}}_{n+1} & =\dot{\mathbf{u}}_{n}\left[(1-\delta) \ddot{\mathbf{u}}_{n}+\delta \ddot{\mathbf{u}}_{n+1}\right] \Delta t, \\
\mathbf{u}_{n+1} & =\mathbf{u}_{n}+\dot{\mathbf{u}}_{n} \Delta t+\left[\left(\frac{1}{2}-\alpha\right) \ddot{\mathbf{u}}_{n}+\alpha \ddot{\mathbf{u}}_{n+1}\right] \Delta t^{2}
\end{aligned}
$$

where $\Delta t=t_{n+1}-t_{n}$ is the time step and $\alpha, \delta$ are integration constants. Depending on the initial values and the displacement $\mathbf{u}_{n+1}$, the acceleration and the velocity at the new point in time can be given as follows:

$$
\begin{aligned}
& \ddot{\mathbf{u}}_{n+1}=a_{0}\left(\mathbf{u}_{n+1}-\mathbf{u}_{n}\right)-a_{2} \dot{\mathbf{u}}_{n}-a_{3} \ddot{\mathbf{u}}_{n}, \\
& \dot{\mathbf{u}}_{n+1}=\dot{\mathbf{u}}_{n}+a_{6} \ddot{\mathbf{u}}_{n}+a_{7} \ddot{\mathbf{u}}_{n+1} .
\end{aligned}
$$

Insertion of these expressions into (24) results in

$$
\begin{gathered}
\left(a_{0} \mathbf{M}+a_{1} \mathbf{C}+\mathbf{K}\right) \mathbf{u}_{n+1} \\
=\mathbf{f}+\mathbf{M}\left(a_{0} \mathbf{u}_{n}+a_{2} \dot{\mathbf{u}}_{n}+a_{3} \ddot{\mathbf{u}}_{n}\right) \\
\quad+\mathbf{C}\left(a_{1} \mathbf{u}_{n}+a_{4} \dot{\mathbf{u}}_{n}+a_{5} \ddot{\mathbf{u}}_{n}\right)
\end{gathered}
$$

containing the Newmark constants:

$$
\begin{aligned}
a_{0} & =\frac{1}{\alpha \Delta t^{2}}, & a_{1} & =\frac{\delta}{\alpha \Delta t}, \\
a_{2} & =\frac{1}{\alpha \Delta t}, & a_{3} & =\frac{1}{2 \alpha}-1, \\
a_{4} & =\frac{\delta}{\alpha}-1, & a_{5} & =\frac{\Delta t}{2}\left(\frac{\delta}{\alpha}-2\right), \\
a_{6} & =\Delta t(1-\delta) \frac{1}{\alpha} & a_{7} & =\delta \Delta t .
\end{aligned}
$$

In (27), only $\mathbf{u}_{n+1}$ is unknown and can be determined by the equilibrium of inner and outer forces. Therewith one obtains the actual velocity $\dot{\mathbf{u}}_{n+1}$ and the acceleration $\ddot{\mathbf{u}}_{n+1}$.

\section{Preparation for the use of boundary elements}

In order to compare results obtained by the use of the FEM and the BEM in this section, the basics of a paper Schanz (2001) are summarized briefly.

As described above, in the original form of the momentum balances of Biot's model (1), partial constitutive laws for the two constituents are used. This form of equations is mostly used in continuum mechanical applications, especially if a clear distinction between microscopic and macroscopic quantities is desired (for a comparison of different representations, see the work of Albers (2010)). But another possible representation has already been shown, namely, the use of total stresses, $\sigma=$ $\sigma^{s}+\sigma^{f} \mathbf{1}$, and the pore pressure, $p$, as variables. Again using Biot's effective stress coefficient $\alpha$ (Biot, 1941) and the solid strain $\epsilon$, the constitutive equation, which is 
equivalent to the above shown effective stress introduced by von Terzaghi modified by the coefficient $\alpha$, is

$$
\sigma=2 G \epsilon+\left(K-\frac{2}{3} G\right)(\operatorname{tr} \epsilon) \mathbf{1}-\alpha p \mathbf{1},
$$

where $G$ and $K$ are the shear modulus and the compressibility modulus of the solid frame, respectively. Here, linear elasticity and small deformations are already accounted for. As explained above, in the case of incompressible constituents, the parameter $\alpha$ is equal to one. Thus, one is left with the classical effective stress concept by von Terzaghi.

A second constitutive law concerns the variation of the fluid volume per unit reference, denoted by $\varsigma$,

$$
\varsigma=\alpha \operatorname{tr} \epsilon+\frac{n^{2}}{R} p,
$$

where $R$ is a material constant (see (3)). Here $\varsigma$ is a kind of strain describing the motion of the fluid relative to the solid. While in Section 1 the deformations are described by the volume changes, $e$ and $\varepsilon$, here instead the solid strain and the variation of fluid volume per unit reference volume, $\epsilon=e$ and $\varsigma$, are used.

Three equations describe the problem. First, there is a mass balance over a reference volume, i.e., the continuity equation

$$
\frac{\partial \varsigma}{\partial t}+\nabla \cdot \mathbf{q}=a,
$$

in which

$$
\mathbf{q}=n \frac{\partial \omega}{\partial t}
$$

is the specific flux depending on the relative displacement $\omega$ of the constituents fluid and solid. The quantity on the right hand side, $a(t)$, is a source term. Even if this source is zero in the present problem, the equations are quoted as they appear in the work of Schanz (2001). The second equation is the momentum balance of the bulk material:

$$
\nabla \cdot \sigma+\mathbf{F}=\rho \frac{\partial^{2} \mathbf{u}}{\partial t^{2}}+n \rho_{f} \frac{\partial^{2} \omega}{\partial t^{2}},
$$

where $\mathbf{F}$ denotes the bulk body force per unit volume and $\mathbf{u}$ the solid displacement. The mass densities are already known (see Eqn. (8)). Here, for the solid strain, the following commonly used form in mechanics is applied:

$$
\epsilon=\frac{1}{2}\left(\nabla \mathbf{u}+\nabla^{T} \mathbf{u}\right)
$$

which implies small deformations. Third, as shown above, the fluid transport is expressed by a generalized Darcy law. Thus,

$$
\mathbf{q}=-\kappa\left(\nabla p+\rho_{f} \frac{\partial^{2} \mathbf{u}}{\partial t^{2}}+\frac{\rho_{a}+n \rho_{f}}{n} \frac{\partial^{2} \omega}{\partial t^{2}}\right),
$$

in which $\kappa$ denotes the permeability. The apparent mass density, $\rho_{a}=C \rho_{f}$, was introduced by Biot (1956) to describe the interaction between fluid and skeleton. The parameter $C$ depends on the pore geometry and on the excitation frequency and is chosen by Schanz (2001) to be 0.66 .

By combination of these equations and elimination of some variables, again a $u$-p-formulation is obtained. A further confirmation that this type of formulation is sufficient for the present approach can be found in the work of Bonnet (1987). It is found in the Laplace domain, denoted by $\mathcal{L}\{f(t)\}=\hat{f}(s)$ with the complex Laplace variable $s$. In this way, from Eqn. (33) Schanz obtains

$$
\hat{\omega}=\underbrace{\frac{\kappa \rho_{f} n^{2} s^{2}}{n^{2} s+s^{2} \kappa\left(\rho_{a}+n \rho_{f}\right)}}_{\beta} \frac{1}{s^{2} n \rho_{f}}\left(\nabla \hat{p}+s^{2} \rho_{f} \hat{\mathbf{u}}\right),
$$

and the final set of differential equations is

$$
\begin{aligned}
& G(\nabla \cdot \nabla) \hat{\mathbf{u}}+\left(K+\frac{1}{3} G\right) \nabla(\nabla \cdot \hat{\mathbf{u}}) \\
&-(\alpha-\beta) \nabla \hat{p}-s^{2}\left(\rho-\beta \rho_{f} \hat{\mathbf{u}}\right)=-\hat{\mathbf{F}}, \\
& \frac{\beta}{s \rho_{f}}(\nabla \cdot \nabla) \hat{p}-\frac{n^{2} s}{R} \hat{p} \\
&-(\alpha-\beta) s \nabla \cdot \hat{\mathbf{u}}=-\hat{a} .
\end{aligned}
$$

Details of the boundary element formulation can be found in the work of Schanz (2001). Here, the approach is presented rather generally: Eqns. (35) and (36) have to be transformed into a boundary integral equation for dynamic poroelasticity. This can be done either using the corresponding reciprocal work theorem (Cheng et al., 1991) or the weighted residual formulation (Dominguez, 1992). These methods need different fundamental solutions, but they both result finally in the same integral equation. Then, a boundary element formulation is achieved by discretizing the boundary surface by iso-parametric elements with polynomial shape functions, by a definition of ansatz functions and by inserting them in the time dependent integral. Next, a temporal discretization has to be carried out. The convolution quadrature method according to Schanz (2001) is the most effective method to achieve this. Thus, a quadrature formula is applied to the integral form of the equation set which results in a boundary element time-stepping formulation. After calculation of integration weights, a system of algebraic relations is obtained to which a direct equation solver is applied.

\section{Comparison of results obtained by the finite and boundary element methods for displacements in a column}

A simple example is investigated using the two numerical methods introduced above. In earlier works it has already been shown that the two-phase model correctly describes 
quasi-static processes like consolidation (Savidis et al., 2011; Rackwitz et al., 2005; Savvidis, 2009). In the present study a dynamic problem is considered which in a similar manner has been investigated by Schanz (2001) using the boundary element method. His results are compared with the current ones obtained using the 3D two phase element developed on the basis of the FEM.

4.1. System. The system considered is built of a column consisting of water saturated Berea sandstone. The width of the column is $b=1 \mathrm{~m}$, the height $h=3 \mathrm{~m}$. The system is shown in Fig. 1. For the FE calculations the height is divided into 10 elements. For Berea sandstone in the work of Schanz (2001) the compressibility modulus $K=8 \cdot 10^{9} \mathrm{~N} / \mathrm{m}^{2}$ and the shear modulus $G=6 \cdot 10^{9}$ $\mathrm{N} / \mathrm{m}^{2}$ are given. From these values there follows Young's modulus to $E_{S}=E=K+\frac{4}{3} G=1.6 \cdot 10^{10} \mathrm{~N} / \mathrm{m}^{2}$, the permeability parameter is $k_{f}=2 \cdot 10^{-6} \mathrm{~m} / \mathrm{s}$, the mass density of the particles is $\rho_{s}=2458 \mathrm{~kg} / \mathrm{m}^{3}$, the mass density of the water is $\rho_{w}=1000 \mathrm{~kg} / \mathrm{m}^{3}$, and the porosity is $n=0.19$. By assuming Poisson's ratio to be $\nu=0$ and by an adequate choice of the boundary conditions the system is investigated as one-dimensional: the soil layer is loaded at the top surface by a distributed load. At this surface the system is permeable while the other boundaries are assumed to be impermeable.

4.2. BEM results. Schanz (2001) investigated the above described column loaded by a Heaviside function with the boundary element method. Further on the problem was calculated analytically by Schanz and Cheng (2000), hints to find an analytical solution were also

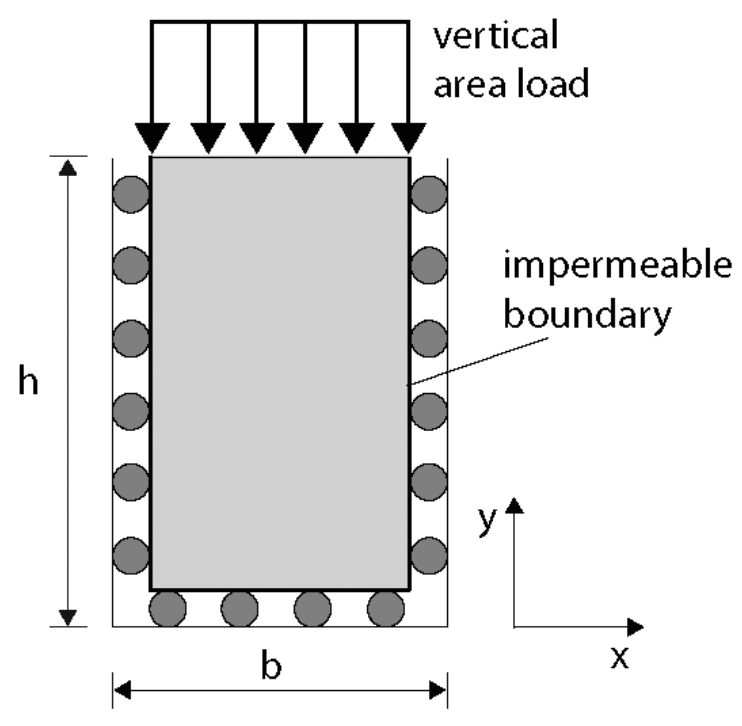

Fig. 1. System for the investigation of the consolidation and the dynamical behavior of a soil layer. given by Eringen and Suhubi (1975). Since this is only possible for simplified cases, the practically impossible case without damping (i.e., no permeability) has been investigated. This means that no relative velocity between the components and no friction between fluid and solid does appear. This unrealistic case is, first, also studied here in order to compare results of the finite element method and of the boundary element method. Afterwards, damping is accounted for. In Fig. 2 the results of the vertical displacements of the upper boundary of the column presented in Fig. 1 are reproduced (after Schanz, 2001). They have been achieved using the boundary element method. The column is loaded from top by a Heaviside function of the force amplitude $1 \mathrm{~N} / \mathrm{m}^{2}$. The authors are aware of the fact that this load is extremely small and unrealistic. However, in order to compare the results of the BEM and the FEM, the value chosen by Schanz (2001) has been retained also for the FEM calculations.

Both panels of Fig. 2 show the progress of the vertical displacements as a function of time. Caused by the load, a wave vertically runs through the column. Already in the short time of $0.01 \mathrm{~s}$ this wave is reflected at the upper and lower boundaries several times. Thereby a zig-zag pattern in the vertical displacement occurs. The amplitude of this displacement is driven by the soil properties and by the size of the load. Schanz (2001) investigated the influence of both, the refinement of the mesh and of the time steps. In the upper panel of Fig. 2] the displacements are given for the meshes of different refinements. Additionally, the analytic solution is presented. It is obvious that the finest mesh (324 linear triangles on 164 nodes) yields the results which fit those of the analytic solution best. Calculations using the coarsest mesh (56 linear triangles on 30 nodes) reveal less accurate results. The coarsening of the mesh yields a numerical damping, i.e., the reflections are expressed less precisely. A similar effect is produced by an enlargement of the time steps. In the bottom panel of Fig. 2 the displacement is illustrated for three time steps of different duration. While for $\Delta t=0.000007 \mathrm{~s}$ (approximately 1428 time steps), the results are nearly in agreement with the analytical solution and also for $\Delta t=0.00001 \mathrm{~s}$ (1000 time steps) only small deviations appear, for $\Delta t=0.0001 \mathrm{~s}$ (100 time steps) the numerical damping becomes clearly evident, which leads to an increasing imprecision of the results.

\subsection{FEM results.}

4.3.1. Number of elements. Calculations of the same type have been carried out for 1000 time steps using the three-dimensional two-phase-model suggested in this contribution. Damping has been neglected by setting the permeability coefficient equal to zero or infinity, $k_{f}=0$ 


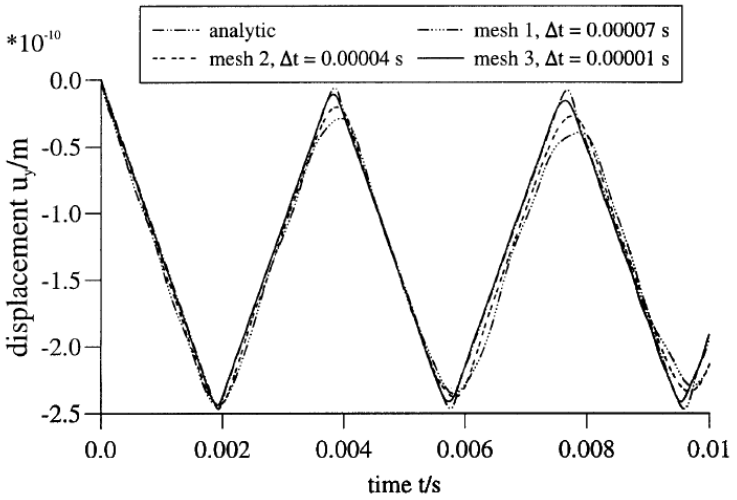

(a)

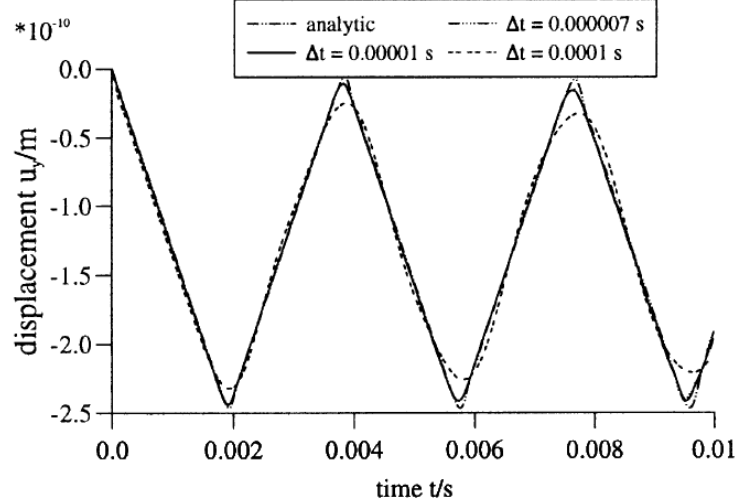

(b)

Fig. 2. Time dependence of the displacements in $y$-direction calculated by the BEM (after Schanz, 2001): influence of the number of elements (a), influence of the number of time steps (b).

or $k_{f} \rightarrow \infty$. Both the limits of the permeability coefficient are unrealistic assumptions for a two-component system and lead to degenerated systems of equations. In both cases, indeed only a one-component system remains. Thus, it is not necessary to use a two-component model. The left panel of Fig. 3 reflects that for $k_{f}=0$, i.e., a completely impermeable material, relatively unstable results are obtained.

Irrespective of the number of elements and for a time step distance (1000 time steps), which later in the paper will be shown to be very well chosen, the results are not satisfying. The fact that the disregard of the diffusion term in calculations of multiphase systems has a destabilizing effect is known in the literature. However, it has been already said that an unrealistic case was investigated and therefore the rather unstable results are not important. The right panel of Fig. 3 shows analogous results using the other limit of the permeability coefficient, $k_{f} \rightarrow \infty$, for which the material is completely permeable. For this value the results are stable and coincide with those for an elastic solid (cf. Fig. 4). In the following it is shown that for realistic cases where diffusion is taken into account the two-phase-element yields physically reasonable results.

It has already been mentioned that for a two-component system without damping, in principle, a solid is present. Therefore, first the column is investigated using a one-component element. As in the work of Schanz (2001), the influence of the number of elements and also of the number of time steps is investigated. The total time of $0.01 \mathrm{~s}$ also used by Schanz (2001) is kept. First, for two different numbers of elements ((a) 4 elements in the $x$-direction, 1 element in the $y$-direction, 10 elements in the $z$-direction, (b) 8 elements in the $x$-direction, 1 element in the $y$-direction, 20 elements in the $z$-direction) the influence of different time steps is investigated. The results are presented in Fig. 4 (left hand side: the coarser mesh, right hand side: the finer mesh).
It is obvious that already the first choice, i.e., the small number of $10 \times 1 \times 4$ elements, is sufficient to describe the problem accurately.

A comparison of the results on the left and right-hand sides of Fig. 4 shows that nearly identical results appear for the two choices of numbers of elements. This shows a contrast of the results which have been obtained by the use of the finite element method with those of Schanz (2001), which have been achieved with the boundary element method.

4.3.2. Time step size. Now the influence of the time step size is investigated. Displacements have been calculated for time steps of $0.001 \mathrm{~s}, 0.0002 \mathrm{~s}, 0.0001 \mathrm{~s}$, $0.00002 \mathrm{~s}$ and $0.00001 \mathrm{~s}$, which is the same as the number of $10,50,100,500$ and 1000 time steps in the total time. Results for 10 time steps are rather disputable. Obviously, this value is too small. For 1000 time steps a result is achieved which is equivalent to the analytic solution (see Fig. 2). The more time steps are used, the better the accordance. For a medium number of time steps, again, the numerical damping appears, i.e., the curves are smoother and the amplitude does not reach the maximum value. The number of elements, in contrast to the number of time steps, is almost irrelevant. In Fig. 5 for the dense time mesh of 1000 time steps, the vertical displacements for five different numbers of elements are shown (30 elements in the $x$-direction, 1 element in the $y$-direction, 12 elements in the $z$-direction; $20 \times 1 \times 8$; $15 \times 1 \times 6 ; 10 \times 1 \times 4$ and $6 \times 1 \times 2)$. Already the coarse number of $6 \times 1 \times 2$ elements yields an acceptable result (see the left panel of Fig. 5). This is confirmed by inspection of the details of the diagram on the right hand side in Fig. 5, Indeed, the results for $6 \times 1 \times 2$ and $10 \times 1 \times 4$ elements slightly deviate from those of the finer mesh, but it is immediately clear that the number of elements is not as important as the discretization in time. 


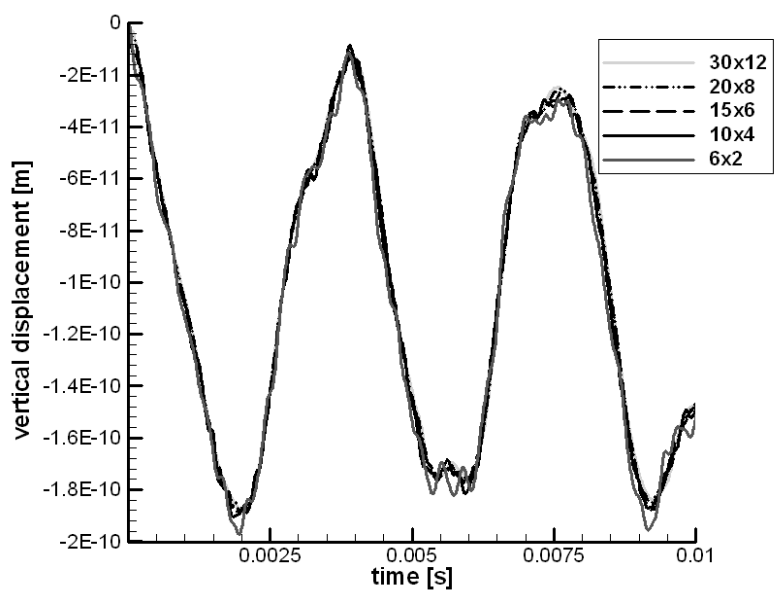

(a)

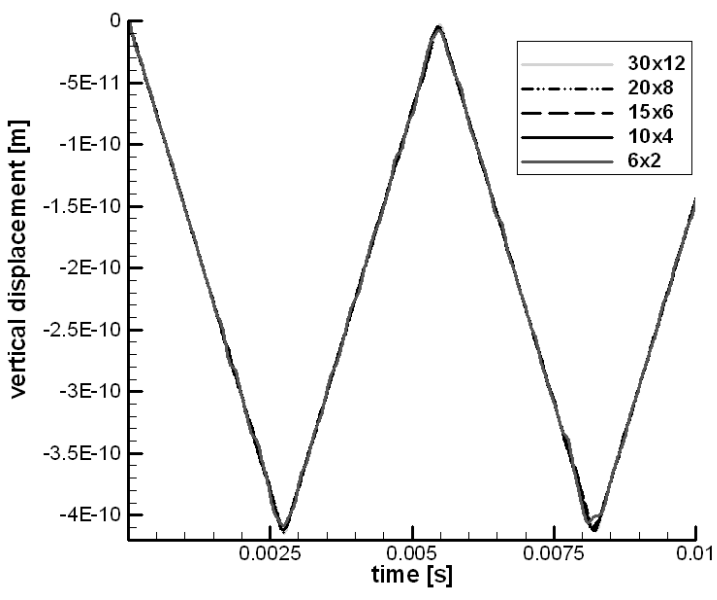

(b)

Fig. 3. Time dependence of the vertical displacements in the column calculated using the two-phase-element for different numbers of elements: permeability coefficient $k_{f}=0,(\mathrm{a}), k_{f} \rightarrow \infty$ (b).

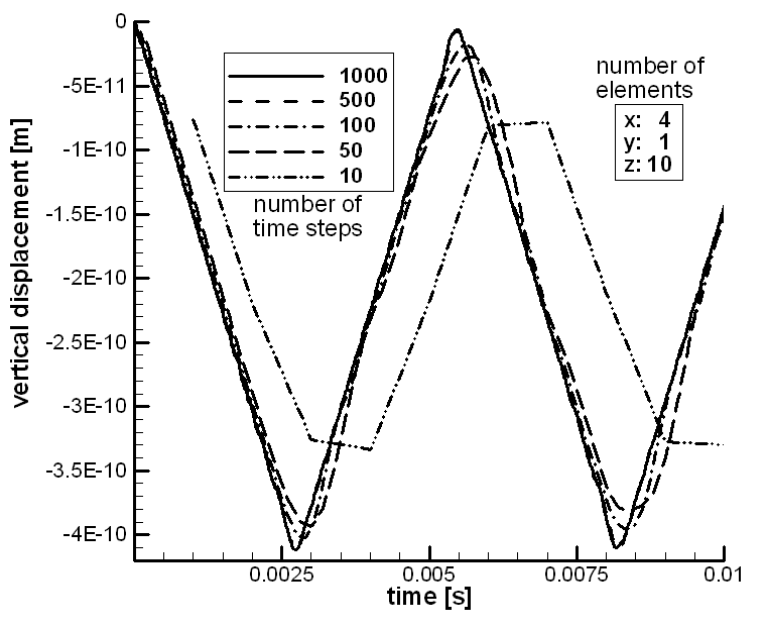

(a)

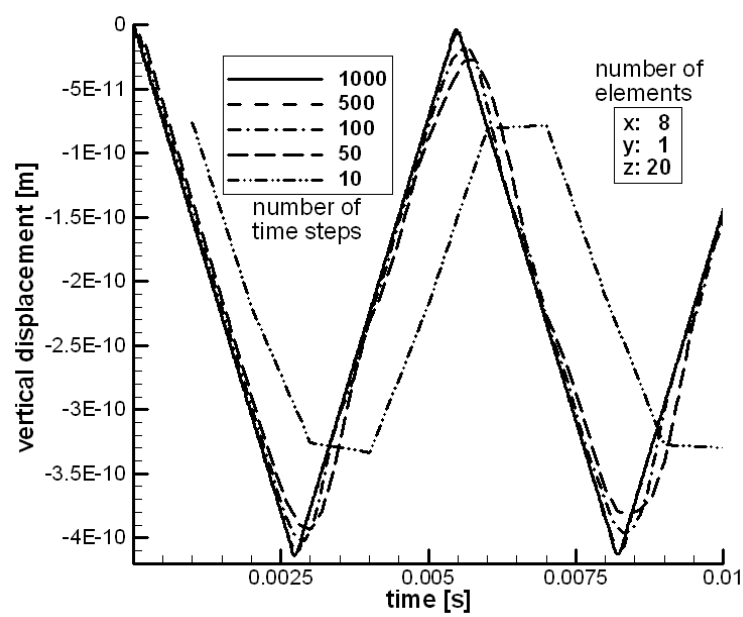

(b)

Fig. 4. Vertical displacements depending on time calculated using a one-phase-element (without damping): $10 \times 1 \times 4$ elements $($ a), $20 \times 1 \times 8$ elements (b)).

4.3.3. Permeability coefficient. Let us come back to the use of the two-phase-element As mentioned above, its use makes only sense if both the diffusion term, i.e., the relative velocity between solid and fluid, and the permeability parameter are taken into account. If the two components appear, in general, they will not have the same velocity such that a diffusion process takes place. Due to friction between the two components, an attenuation appears (not to compare with the above mentioned numerical damping which arises because of an inadequate number of elements or time steps). This means that a wave which is generated by a Heaviside load is further reflected on the upper and lower boundaries of the column. However, its amplitude is decreasing in time. This is illustrated in Fig.6.

The left hand side shows the result of equivalent calculations as they were needed to obtain the results of the left hand side of Fig. 5 but instead of the one-phase-element here the two-phase-element with the permeability coefficient $k_{f}=10^{-2} \mathrm{~m} / \mathrm{s}$ is used. It is obvious that, with this element, results of the same quality are obtained as with the one-phase-element. This is, again, confirmed by the detail on the right-hand side of Fig. 6. Also here, only the calculations using $6 \times 1 \times 2$ and $10 \times 1 \times 4$ elements are slightly deviating from the exact solution. Thus, also for the two-phase-element the number of elements is not of the same importance as for the boundary element method.

The influence of the attenuation (friction between the two components, solid and fluid), and thus of the value of the permeability coefficient $k_{f}$ on the quality of the results, is presented in Fig. 7. The vertical displacements 


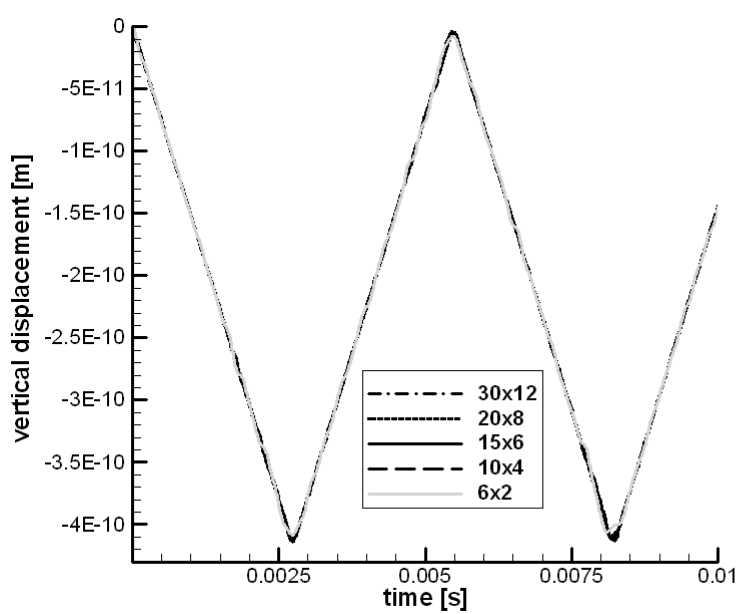

(a)

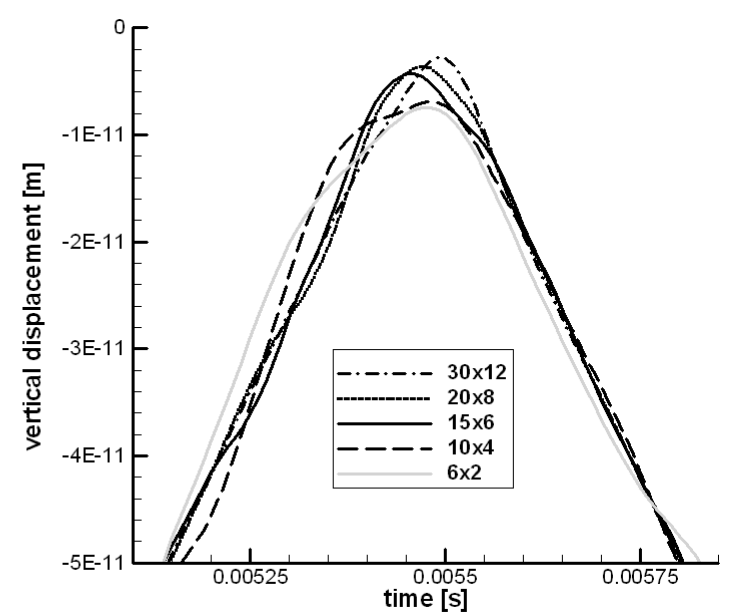

(b)

Fig. 5. Vertical displacements in dependence on time calculated using the two-phase-element $\left(k_{f} \rightarrow \infty\right)$. 1000 time steps, a different number of elements ((b) is a detail of (a)).

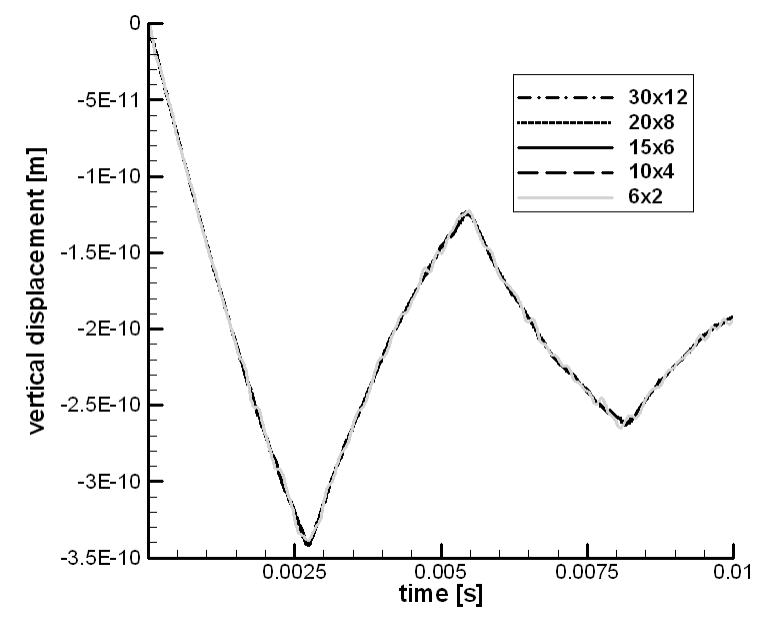

(a)

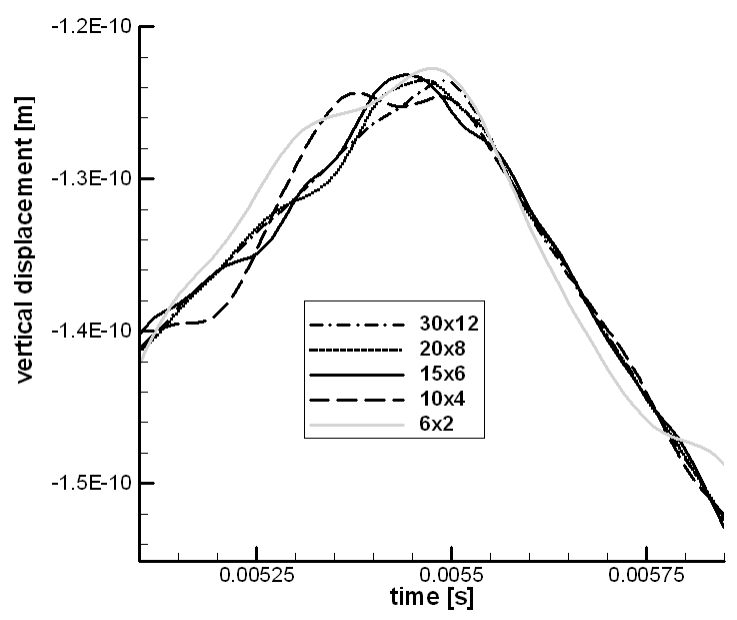

(b)

Fig. 6. Vertical displacements depending on time calculated using the two-phase-element $\left(k_{f}=10^{-2} \mathrm{~m} / \mathrm{s}\right) .1000$ time steps, different numbers of elements ((b) is a detail of (a)).

are much smaller if the diffusion is not taken into account. The incorporation of the diffusion yields more stable numerical results.

4.3.4. Pore pressure. Even though for the current project the displacements are of main interest, for the sake of completeness, also a qualitative comparison of the second important quantity appearing in porous media, the pore pressure, is shown.

From Fig. 8 it is obvious that the results for the pore pressure for FEM and BEM calculations are qualitatively the same. For a high number of elements in the case of the FEM or a fine mesh for the BEM and a high number of time steps, the results are stable and sufficiently close to the analytical solution (see Schanz and Cheng, 2000) which is presented on the right hand side of Fig. 8 . While
Schanz (2001) reports on only slight dependence on the time step size for the BEM, for the FEM it is obvious that the reduction of the time step size yields bigger deviations from the stable solution than a reduction of the number of elements. The deflections from the stable solution of the dotted curve on the left-hand side of Fig. 8 , standing for a small number of elements, are significantly weaker than those of the dash-dotted line representing the lowest number of time steps (100 time steps in $0.01 \mathrm{~s}$ ).

\section{Final remarks}

In this work the displacements occurring in a column consisting of porous material which is vertically loaded from the upper boundary by a Heaviside area load have been investigated. Results, on the one hand obtained by 


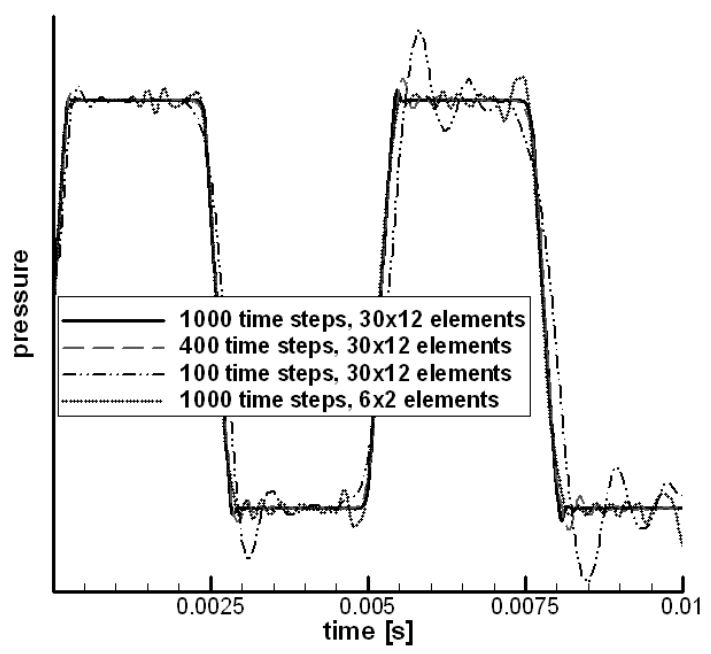

(a)

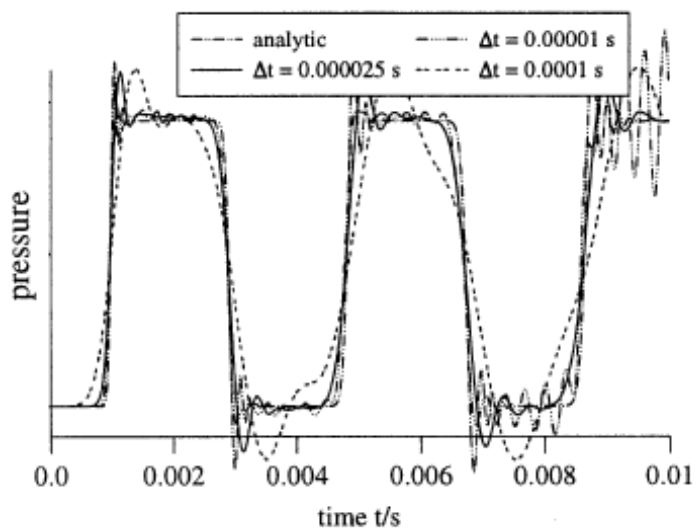

(b)

Fig. 8. Pore pressure at the lower part of the porous column: FEM results for different numbers of time steps for a fine spatial discretization, the dotted line represents very few elements for a high number of time steps (a), BEM results by Schanz (2001) for a fine mesh and the same number of time steps as presented in the left panel $(\Delta t=0.00001 \mathrm{~s} \rightarrow 1000$ time steps, $\Delta t=0.000025 \mathrm{~s} \rightarrow 400$ time steps, $\Delta t=0.0001 \mathrm{~s} \rightarrow 100$ time steps) (b).

the use of the finite element method and on the other with the boundary element method, are compared. The influence of the number of elements and the time step size, as well as the impact of different values of the permeability coefficient, have been studied, leading to the following conclusions:

- The choice of a higher number of elements is, at least for this example, more important for the BEM than for the FEM in order to obtain accurate results. For the FEM calculations, already a relatively coarse mesh yielded smooth results, while for the BEM calculations a rather fine mesh was necessary.

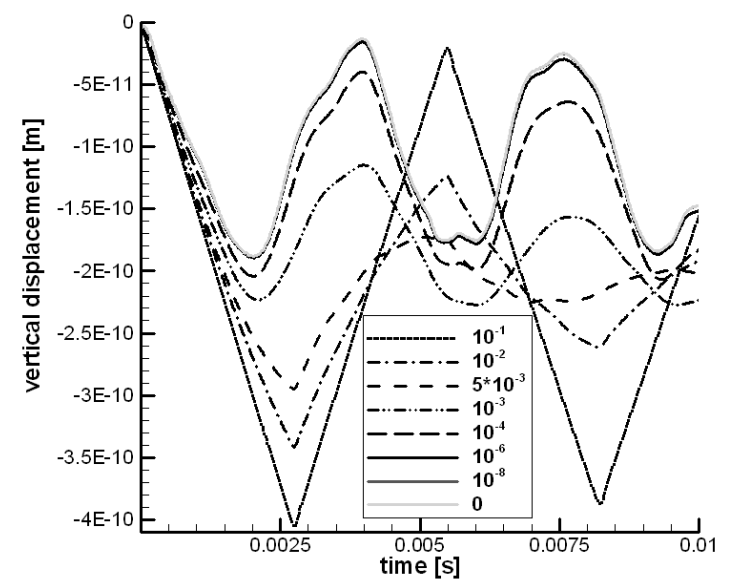

Fig. 7. Vertical displacements depending on time calculated using the two-phase-element for different values of the permeability parameter $k_{f}[\mathrm{~m} / \mathrm{s}] ; 1000$ time steps.
- In the case of both approaches, the number of time steps in a given total time is, with respect to the accuracy of the results, of significant importance. The more time steps are used, the closer the result to the analytical solution. For a smaller number of elements in both the approaches numerical damping becomes evident, i.e., imprecise results are obtained and the amplitude does not reach the maximum value of the analytical solution.

- The amplitude of the vertical displacements is much larger if the permeability parameter is taken into account. For higher values of this parameter the numerical results are more stable.

These results show that the sound propagation through porous soils can be studied more or less equivalently with the use of both numerical methods, namely, the FEM and the BEM. The reason for the choice of either of the methods, thus, will not be the accuracy of the results but the advantages of one or the other method concerning the keeping of boundary conditions or the reduction of calculation time. In fact, the BEM has the advantage to fulfil the Sommerfeld radiation condition automatically while it has the disadvantage of possessing fully occupied, non-symmetric, complex matrices leading to longer computation times.

\section{Acknowledgment}

The authors appreciate very much the financial support of the grants SA 310/25 and ES 70/5 from the German Research Foundation (DFG). 


\section{References}

Albers, B. (2010). Modeling and Numerical Analysis of Wave Propagation in Saturated and Partially Saturated Porous Media, Postdoctoral thesis, Veröffentlichungen des Grundbauinstitutes der Technischen Universität Berlin, Vol. 48, Shaker, Aachen.

Allard, J.F. (1993). Propagation of Sound in Porous Media. Modelling Sound Absorbing Materials, Elsevier, Essex.

Atalla, N., Hamdi, A.M. and Panneton, R. (2001). Enhanced weak integral formulation for mixed (u,p) poroelastic equations, Journal of the Acoustical Society of America 109(6): 3065-3068.

Atalla, N., Panneton, R. and Debergue, P. (1998). A mixed pressure-displacement formulation for poroelastic materials, Journal of the Acoustical Society of America 104(3): 1444-1452.

Biot, M.A. (1941). General theory of three dimensional consolidation, Journal of Applied Physics 12(2): 155-164.

Biot, M.A. (1956). Theory of propagation of elastic waves in a fluid saturated porous solid, I: Low frequency range, II: Higher frequency range, Journal of the Acoustical Society of America 28(2): 168-191.

Bonnet, G. (1987). Basic singular solutions for a poroelastic medium in the dynamic range, Journal of the Acoustical Society of America 82(5): 1758-1762.

Cheng, A.H.-D., Badmus, T. and Beskos, D.E. (1991). Integral equations for dynamic poroelasticity in frequency domain with BEM solution, Journal of Engineering Mechanics (ASCE) 117(5): 1136-1157.

de Boer, R. and Ehlers, W. (1986). Theorie der Mehrkomponentenkontinua mit Anwendung auf bodenmechanische Probleme, Teil I, Technical Report 40, Forschungsberichte aus dem Fachbereich Bauwesen der Universität-GH Essen, Essen.

Dominguez, J. (1992). Boundary element approach for dynamic poroelastic problems, International Journal for Numerical Methods in Engineering 35(2): 307-324.

Eringen, A.C. and Suhubi, S.S. (1975). Elastodynamics, Vol. II, Academic Press, New York, NY.

Fischer, M. (2004). The Fast Multipole Boundary Element Method and its Application to Structure-Acoustic Field Interaction, Ph.D. thesis, Universität Stuttgart, Stuttgart.

Goodman, M.A. and Cowin, S.C. (1972). A continuum theory of granular materials, Archive for Rational Mechanics and Analysis 44(4): 249-266.

Göransson, P. (1995). A weighted residual formulation of the acoustic wave propagation through a flexible porous material and comparison with a limp material model, Journal of Sound and Vibration 182(3): 479-494.

Hild, P. (2011). A sign preserving mixed finite element approximation for contact problems, International Journal of Applied Mathematics and Computer Science 21(3): 487-498, DOI: 10.2478/v10006-011-0037-7.
Holler, S. (2006). Dynamisches Mehrphasenmodell mit hypoplastischer Materialformulierung der Feststoffphase, Ph.D. thesis, RWTH Aachen, Aachen.

Kelder, O. and Smeulders, D.M.J. (1997). Observation of the Biot slow wave in water-saturated Nivelsteiner sandstone, Geophysics 62(6): 1794-1796.

Kogut, J. and Ciurej, H. (2010). A vehicle-track-soil dynamic interaction problem in sequential and parallel formulation, International Journal of Applied Mathematics and Computer Science 20(2): 295-303, DOI: 10.2478/v10006-010-0022-6.

Korsawe, J. and Starke, G. (2005). A least-squares mixed finite element method for Biot's consolidation problem in porous media, SIAM Journal on Numerical Analysis 43(1): 318-339.

Korsawe, J., Starke, G., Wang, W. and Kolditz, O. (2006). Finite element analysis of poro-elastic consolidation in porous media: Standard and mixed approaches, Computer Methods in Applied Mechanics and Engineering 195(9-12): 1096-1115.

Lewis, R.W. and Schrefler, B.A. (1998). The Finite Element Method in the Static and Dynamic Deformation and Consolidation of Porous Media, Wiley, Chichester.

Naumann, K. (2004). Implementierung eines Finiten Elementes in das FEM-Programmsystem ANSYS zur gekoppelten Fluid-Struktur Berechnung poröser Medien, Master's thesis, TU Berlin, Berlin.

Panneton, R. and Atalla, N. (1997). An efficient finite element scheme for solving the threedimensional poroelasticity problem in acoustics, Journal of the Acoustical Society of America 101(6): 3287-3298.

Plona, T. J. (1980). Observation of a second bulk compressional wave in a porous medium at ultrasonic frequencies, $A p$ plied Physics Letters 36(4): 259-261.

Rackwitz, F., Naumann, K. and Savidis, S.A. (2005). Implementierung eines Finiten Elements zur Konsolidationsberechnung mit ANSYS, 23rd CADFEM Users' Meeting 2005, Bonn, Germany, (on CD-ROM/DVD).

Savidis, S.A., Albers, B., Taşan, H.E. and Savvidis, G. (2011). Finite-Elemente-Berechnungen quasistatischer und dynamischer Probleme mit einem poroelastischen Zweikomponentenmodell, Bauingenieur 5: 241-249.

Savvidis, G. (2009). Implementierung eines Finiten Elements in das FEM-Programmsystem ANSYS zur gekoppelten Fluid-Struktur Berechnung von wassergesättigten Böden, Master's thesis, TU Berlin, Berlin.

Schanz, M. (2001). Application of 3d time domain boundary element formulation to wave propagation in poroelastic solids, Engineering Analysis with Boundary Elements 25(4-5): 363-376.

Schanz, M. and Cheng, A.H.-D. (2000). Transient wave propagation in a one-dimensional poroelastic column, Acta Mechanica 145(1-4): 1-18. 
Schrefler, B.A. and Scotta, R. (2001). A fully coupled dynamic model for two-phase fluid flow in deformable porous media, Computer Methods in Applied Mechanics and Engineering 190(24-25): 3223-3246.

Taşan, H.E. (2012). Zur Dimensionierung der MonopileGründungen von Offshore-Windenergieanlagen, Ph.D. thesis, Veröffentlichungen des Grundbauinstitutes der Technischen Universität Berlin, Vol. 52, Aachen.

Taşan, H.E., Rackwitz, F. and Savidis, S.A. (2010). Behaviour of cyclic laterally loaded diameter monopiles in saturated sand, Proceedings of the 7th European Conference of $\mathrm{Nu}$ merical Methods in Geotechnical Engineering, Trondheim, Norway, pp. 889-894.

von Estorff, O. and Hagen, C. (2006). Iterative coupling of FEM and BEM in 3D transient elastodynamics, Engineering Analysis with Boundary Elements 30(7): 611-622.

von Terzaghi, K. (1936). The shearing resistance of saturated soils and the angle between the planes of shear, $1 \mathrm{st}$ International Conference on Soil Mechanics and Foundation Engineering, Cambridge, MA, USA, Vol. 1, pp. 54-56.

Wilmanski, K. (1996). Porous media at finite strains-The new model with the balance equation for porosity, Archives of Mechanics 48(4): 591-628.

Zienkiewicz, O.C., Chan, A.H.C., Pastor, M., Schrefler, B.A. and Shiomi, T. (1999). Computational Geomechanics with Special Reference to Earthquake Engineering, John Wiley $\&$ Sons, West Sussex.

Zienkiewicz, O.C. and Shiomi, T. (1984). Dynamic behaviour of saturated porous media: The generalized Biot formulation and its numerical solution, International Journal for Numerical and Analytical Methods in Geomechanics 8(1): 71-96.

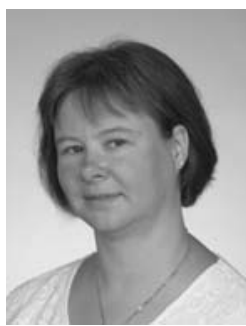

Bettina Albers received her doctoral degree for a work on adsorption/diffusion problems in porous media from the Berlin Institute of Technology (TUB) in 2000. In 2010 she finished her habilitation in mechanics in the same university with a work on modeling and numerical analysis of wave propagation in saturated and partially saturated porous media. Recently, she has been awarded an Einstein Junior Fellowship by the Einstein Foundation in Berlin. The topic of this research for three years will be the investigation of the influence of the hysteretic behavior of the capillary pressure on the propagation of sound waves in partially saturated porous media.

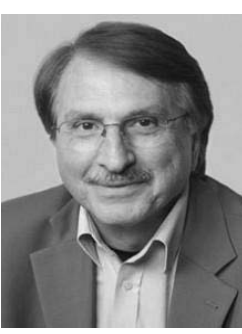

Stavros A. Savidis is a professor of geotechnical engineering at the Berlin Institute of Technology (TUB) and the head of the Institute of Geotechnical Engineering and Soil Mechanics. He obtained his doctoral degree in geotechnical engineering in 1971. In 1977 he finished his habilitation in the same university. From 1983 to 1987 he was the rector of the Democritus University of Thrace (Greece). Since 1987 he has been a professor at TUB. He is also a partner in the engineering company GuD (Geotechnics and Dynamics Consult) in Berlin and in Hanseatic Power Cert in Hamburg.

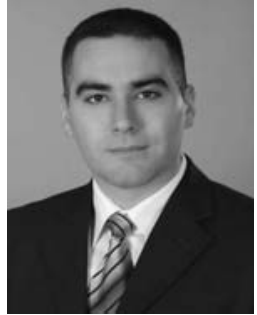

H. Ercan Tasan received his doctoral degree in offshore foundation engineering from the Berlin Institute of Technology (TUB) in 2011. He is the chief executive officer of the company TRADYNA, which is engaged in special problem statements of engineering and construction, and is distinguished by the recent know-how from research and development works. His current research interests include new innovative foundations for offshore structures and design of cyclic loaded offshore pile foundations.

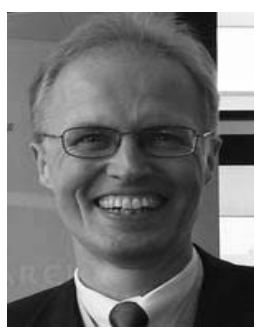

Otto von Estorff is a professor of engineering mechanics at the Hamburg University of Technology (TUHH) and the head of the Institute of Modelling and Computation. He obtained his doctoral degree in engineering mechanics in 1986. After one year of teaching and research at the MIT, Boston, he joined IABG, a German consulting company, where he was responsible for numerous national and international projects in the fields of computational methods and acoustics. In 1997 he accepted a call to become a professor at TUHH. Currently, he is the president of the German Acoustical Society, the presiden of NIT, Hamburg, and the CEO of Novicos, Hamburg.

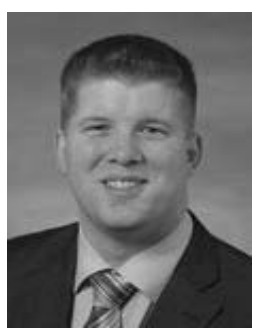

Malte Gehlken has studied theoretical mechanical engineering at the Hamburg University of Technology. Since 2009 he has been employed there as a research fellow at the Institute of Modelling and Computation. In his research he is concerned with numerical simulations in the field of acoustics as well as fast solution procedures for the boundary element method.

\section{Appendix}

\section{Index notation of some equations}

In Sections 1.3 and 1.4 we have taken over the formulation used by Zienkiewicz et al. (1999). Since this notation in absolute notation is at some places unclear we add the index notation of some equations in this appendix.

$$
\begin{aligned}
\text { (5) } \rightarrow & \sigma_{i j}=\sigma_{i j}^{\prime}-\alpha \delta_{i j} p, \\
\text { (6) } \rightarrow & \text { engineering strains } \\
& d \varepsilon_{i}=d u_{i, i} \text { (with the repeated index not summed), } \\
\text { (71) } \rightarrow & \sigma_{i j, j}-\rho \ddot{u}_{i}-\rho_{f}\left[\dot{w}_{i}+w_{j} w_{i, j}\right]+\rho b_{i}=0, \\
\text { (10) } \rightarrow & -p_{, i}-R_{i}-\rho_{f} \ddot{u}_{i}-\rho_{f}\left[\dot{w}_{i}+w_{j} w_{i, j}\right] / n \\
& +\rho b_{i}=0, \quad w_{i}=k_{i j} R_{j}, \\
\text { (11) } \rightarrow & w_{i}=k\left(-p_{, i}+\rho b_{i}\right), \\
\text { (12) } \rightarrow & w_{i, i}+\alpha \dot{\varepsilon}_{i i}+\frac{\dot{p}}{Q}+n \frac{\dot{\rho}_{f}}{\rho_{f}}+\dot{s}_{0}, \\
\text { (13) } \rightarrow & \left(k_{i j}\left(-p_{, j}+\rho b_{j}\right)\right)_{, i}+\alpha \dot{\varepsilon}_{i i} .
\end{aligned}
$$

Received: 26 October 2011 Revised: 4 April 2012 Re-revised: 4 June 2012 